\title{
A Patient with Hepatocellular Carcinoma with Isolated Right Atrial Metastases
}

\author{
Hiroaki Takaya ${ }^{1}$, Hideto Kawaratani ${ }^{1}$, Kenichiro Seki ${ }^{1}$, Yasushi Okura ${ }^{2}$, Mitsuteru Kitade ${ }^{1}$, \\ Tadashi Namisaki ${ }^{1}$, Masayoshi Sawai ${ }^{1}$, Yasuhiko Sawada ${ }^{1}$, Takuya Kubo ${ }^{1}$, Akira Mitoro ${ }^{1}$, \\ Junichi Yamao $^{2}$ and Hitoshi Yoshiji ${ }^{1}$
}

\begin{abstract}
:
Hepatocellular carcinoma (HCC) with isolated right atrial metastasis is extremely rare; most cases are considered inoperable. We herein report the case of a 74-year-old man with HCC with isolated right atrial metastases without hepatic vein invasion; the right atrial lesion was resected because of the risk of heart failure and sudden death. Postoperatively, he underwent transcatheter arterial chemoembolization and radiofrequency ablation for intrahepatic HCC. He recovered completely, with a long-term survival of 36 months. This is the first report of an HCC patient with isolated right atrial metastases without hepatic vein invasion. Tumorectomy for solitary atrial metastasis is effective for HCC patients.
\end{abstract}

Key words: hepatocellular carcinoma, right atrial metastases, transcatheter arterial chemoembolization, radiofrequency ablation, chronic hepatitis type $\mathrm{C}$

(Intern Med 56: 2589-2594, 2017)

(DOI: 10.2169/internalmedicine.8568-16)

\section{Introduction}

It is well known that patients with liver cirrhosis develop hepatocellular carcinoma (HCC) with high frequency (1). Recent advances in diagnostic imaging have enabled the detection of HCC in the early stages. However, HCC is usually detected only at advanced stages because of the lack of associated symptoms at earlier stages. Vascular invasion is a major prognostic factor for HCC. However, cases with right atrial invasion of HCC are rare $(0.67-4.1 \%)(2-4)$, and the occurrence of metastasis to the right atrium without hepatic vein invasion is even rarer. Most such patients are considered inoperable because of their advanced disease stage and poor general condition. However, such cases should undergo surgery because of the associated risk of sudden death from right heart failure and pulmonary embolism $(5,6)$.

We herein report a rare case of a patient having HCC with right atrial metastases without hepatic vein invasion. The patient underwent operation for right atrial metastases and subsequent transcatheter arterial chemoembolization
(TACE) and radiofrequency ablation (RFA) for intrahepatic HCC.

\section{Case Report}

A 74-year-old man was referred to our hospital for the further examination of cardiac and hepatic tumors. He had been diagnosed with chronic hepatitis type $\mathrm{C}$ for seven years. He had no history of habitual smoking or alcohol intake and had no family history of cardiac or liver disease. He had never received a blood transfusion. After the diagnosis, he received ursodeoxycholic acid but never received interferon or direct-acting antiviral therapy. He underwent a blood examination every 2-3 months. His transaminase levels were well controlled $(<30 \mathrm{IU} / \mathrm{L})$, but his platelet levels were low $\left(6.5 \times 10^{4} / \mu \mathrm{L}\right)$. His Child-Pugh score was 5 points. He underwent enhanced computed tomography (CT) or ultrasonography at regular intervals. Esophagogastroduodenoscopy revealed no esophago-gastric varices, and his Eastern Cooperative Oncology Group performance status was 0 . Furthermore, he had received abdominal enhanced CT at 4-

${ }^{1}$ Third Department of Internal Medicine, Nara Medical University, Japan and ${ }^{2}$ Department of Endoscopy, Nara Medical University, Japan Received: November 15, 2016; Accepted: February 20, 2017; Advance Publication by J-STAGE: September 6, 2017 Correspondence to Dr. Hideto Kawaratani, kawara@naramed-u.ac.jp 
(A)

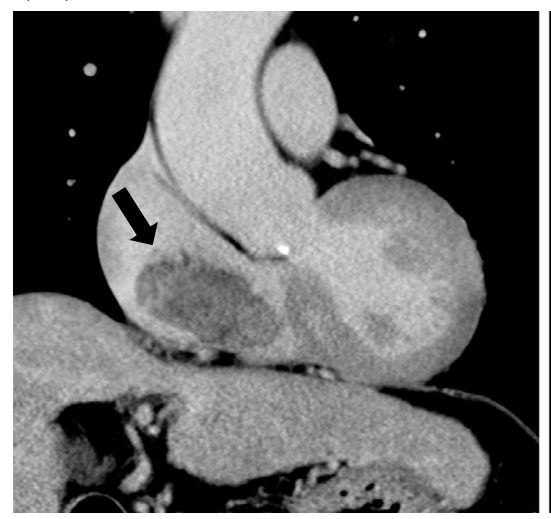

(B)

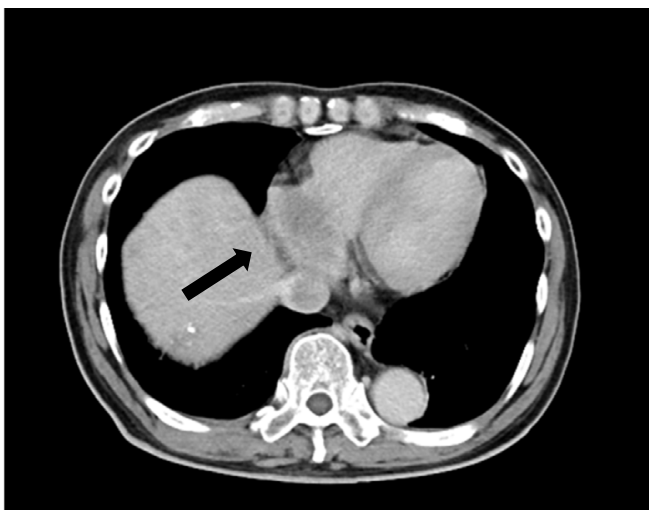

Figure 1. Computed tomography of the chest. (A) The 4.4-cm right atrial tumor (arrow) extended into the proximal inferior vena cava. (B) The cardiac tumor (arrow) did not connect to the hepatic vein or hepatic tumor.

(A)

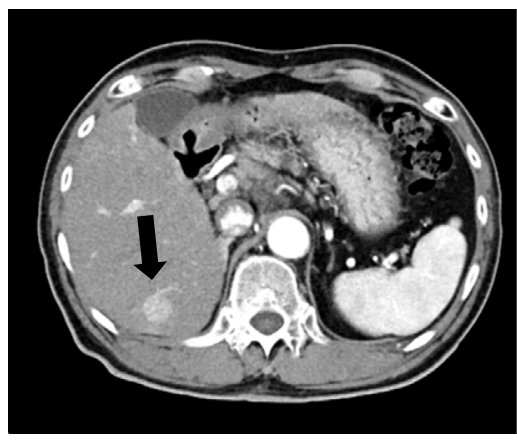

(B)

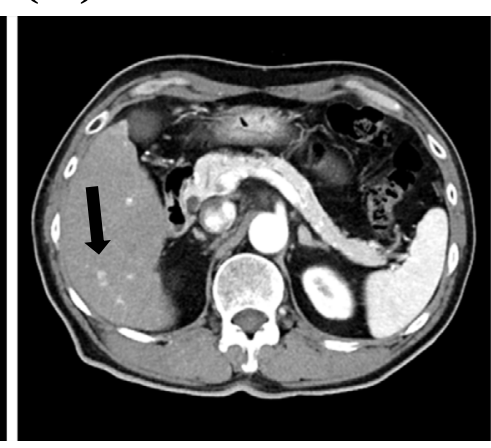

(C)

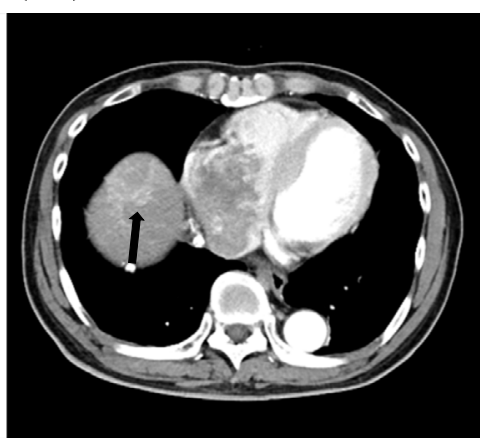

Figure 2. Abdominal computed tomography. (A, B, and C) Three hypervascular tumors (arrows: one 1.8 -cm tumor and one $1.0-\mathrm{cm}$ tumor in segment 6 and one 1.0-cm tumor in segment 8) with a rapid washout pattern in the liver can be observed.

month intervals for 1.5 years because of high levels of protein induced by Vitamin $\mathrm{K}$ absence or antagonist (PIVKA)II (approximately $200 \mathrm{mAU} / \mathrm{mL}$ ). Follow-up enhanced CT revealed three hypervascular hepatic masses with a rapid washout pattern in liver segments 6 and 8 (one 1.8-cm mass and one 1.0-cm mass in segment 6 and one 1.0-cm mass in segment 8 ) and a $4.4-\mathrm{cm}$ tumor in the right atrium extending into the proximal inferior vena cava (IVC) (Fig. 1, 2). The levels of PIVKA-II were further increased from approximately 200 to $421 \mathrm{mAU} / \mathrm{mL}$ (Table). HCC of segment 6 had access to the intrahepatic vein but had not invaded it, and no metastasis to other organs was found.

His electrocardiogram and chest X-ray findings were normal. Echocardiography revealed a $4.4-\mathrm{cm}$ low-echoic pedunculated mass in the right atrium, which was attached to the right atrium wall and could be easily moved. Magnetic resonance imaging (MRI) revealed low intensity on T1-weighted images, slightly high intensity on T2-weighted images, and enhancement in the arterial and hepatobiliary phases on gadolinium-ethoxybenzyl-diethylenetriaminepentaacetic acidenhanced MRI. We could not determine whether or not the right atrial tumor represented HCC metastasis. However, a biopsy suggested an associated risk of heart failure, sudden death, and metastasis. Cardiac tumorectomy of the right atrium was therefore performed. The $4.4-\mathrm{cm}$ tumor was located on the right atrium (Fig. 3). On a histological examination, the tumor had atypical cells with a cord-like structure, globular hyaline bodies, and clear cells. The tumor had invaded into the right atrial endocardium (Fig. 4). On immunohistochemical staining, cytokeratin (CAM) 5.2 and hepatocyte-specific antigen (HSA) were positive, and AE1/ AE3 was negative (Fig. 5). Therefore, we diagnosed the right atrial tumor to be $\mathrm{HCC}$ metastasis with poor differentiation.

After the operation, he underwent TACE and RFA for intrahepatic HCC. He recovered completely, and his levels of PIVKA-II decreased after the treatment (50-70 mAU/mL). After six months, he developed lung metastasis of HCC without intrahepatic HCC. He underwent chemotherapy with sorafenib. The lung metastasis stabilized, and he had a longterm survival of 36 months without intrahepatic HCC. He had no brain metastasis, but ultimately died of brainstem 
Table. Laboratory Data on Admission.

\begin{tabular}{lrlr}
\hline Peipheral Blood & & Lactate dehydrogenase & $506 \mathrm{IU} / \mathrm{L}$ \\
White blood cell & $5,600 / \mu \mathrm{L}$ & Serum total bilirubin & $1.0 \mathrm{mg} / \mathrm{dL}$ \\
Red blood cell & $470 \times 10^{4} / \mu \mathrm{L}$ & Blood urea nitrogen & $23 \mathrm{mg} / \mathrm{dL}$ \\
Hemoglobin & $15.9 \mathrm{~g} / \mathrm{dL}$ & Serum creatinine & $0.81 \mathrm{mg} / \mathrm{dL}$ \\
Hematocrit & $46.1 \%$ & Serum sodium & $138 \mathrm{mEq} / \mathrm{L}$ \\
Platelet & $6.5 \times 10^{4} / \mu \mathrm{L}$ & Serum potassium & $4.3 \mathrm{mEq} / \mathrm{L}$ \\
& & Serum chloride & $103 \mathrm{mEq} / \mathrm{L}$ \\
Blood Coagulation & & Serum calcium & $9.3 \mathrm{mg} / \mathrm{dL}$ \\
Prothrombin time (INR) & 1.13 & Fasting blood glucose & $134 \mathrm{mg} / \mathrm{dL}$ \\
Prothrombin time (\%) & $76 \%$ & & \\
Activated partial thromboplastin time & $35.8 \mathrm{sec}$ & Tumor Marker & \\
& & AFP & $16.6 \mathrm{ng} / \mathrm{mL}$ \\
Blood Chemistry & & AFP(L3) & $6.7 \%$ \\
C-reactive protein & & PIVKA-II \\
Total protein & $7.6 \mathrm{gg} / \mathrm{dL}$ & CEA & $421 \mathrm{mAU} / \mathrm{mL}$ \\
Serum albumin & $3.9 \mathrm{~g} / \mathrm{dL}$ & CA19-9 & $4.0 \mathrm{ng} / \mathrm{mL}$ \\
Aspartate aminotransferase & $68 \mathrm{IU} / \mathrm{L}$ & & $40 \mathrm{U} / \mathrm{mL}$ \\
Alanine aminotransferase & $65 \mathrm{IU} / \mathrm{L}$ & Viral Infection & \\
Alkaline phosphatase & $178 \mathrm{IU} / \mathrm{L}$ & HBs antigen & negative \\
$\gamma$-glutanyl transpeptidase & $23 \mathrm{IU} / \mathrm{L}$ & HCV antibody & $\mathrm{positive}$ \\
\hline
\end{tabular}

INR: International Normalized Ratio

(A)

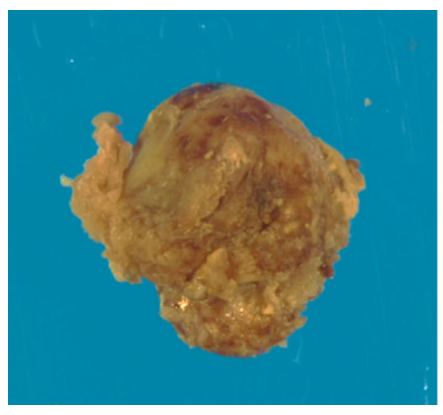

\section{(B)}

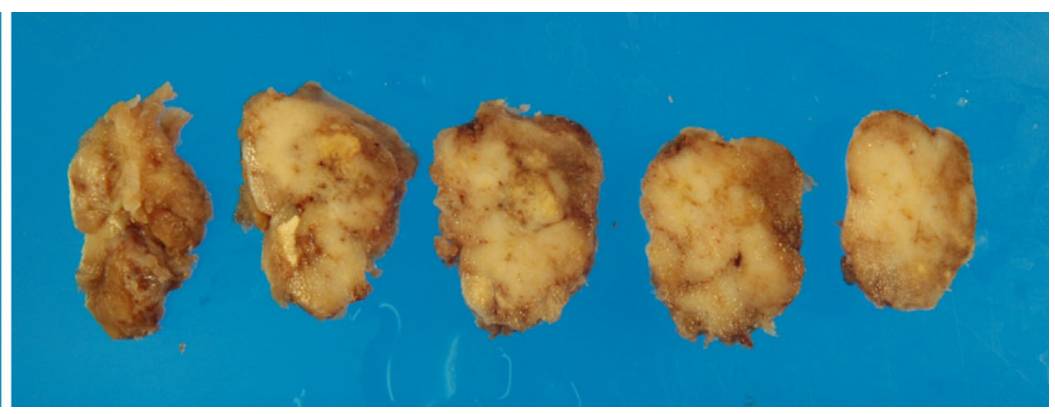

Figure 3. The extracorporeal circulation was operated upon after median sternotomy. Right atriotomy revealed the invasion of a soft-tissue tumor gray-yellow in color in the right atrium. It was completely removed by cardiac tumorectomy. (A) The resected specimen of the right atrium. (B) Cross-sectional slices of the tumor.

hemorrhaging.

\section{Discussion}

Cases of cardiac tumor are rare, reported to occur in $0.0017-0.28 \%$ of patients on an autopsy (7). Approximately $70 \%$ of cardiac tumors are benign, and the remaining are malignant. Malignant cardiac tumors (primary and metastasis) occur more frequently in the right heart system than in the left heart system. Metastatic cardiac tumors develop 6-40 times more frequently than primary tumors and usually occur in the myocardium, followed by the epicardium, pericardium, and endocardium (7-9). Most metastatic cardiac tumors originate from lung cancers, lymphomas, or breast cancers (9), but those resulting from gastrointestinal cancer or HCC are rare (10).

Kuratomi et al. and Miyake et al. reported that $\mathrm{HCC}$ mainly invades the liver portal system (and liver cirrhosis frequently involves arterio-portal shunt in the liver) $(11,12)$. Based on these reports, the cardiac invasion of HCC is considered to be rare. Right atrial invasion has been reported in approximately $0.67-4.1 \%$ of patients who underwent HCC autopsy (2-4). Furthermore, HCC with isolated right atrial metastases without hepatic vein invasion were reported in $0.41 \%$ of patients who underwent HCC autopsy (2). It is considered that metastatic cardiac tumors spread through the coronary artery or IVC, by direct invasion, or through lymphatic vessels. There are few reports $(5,13)$ of HCC with right atrial metastases and tumor thrombus extending through the major hepatic veins and IVC. In the present 
(A)

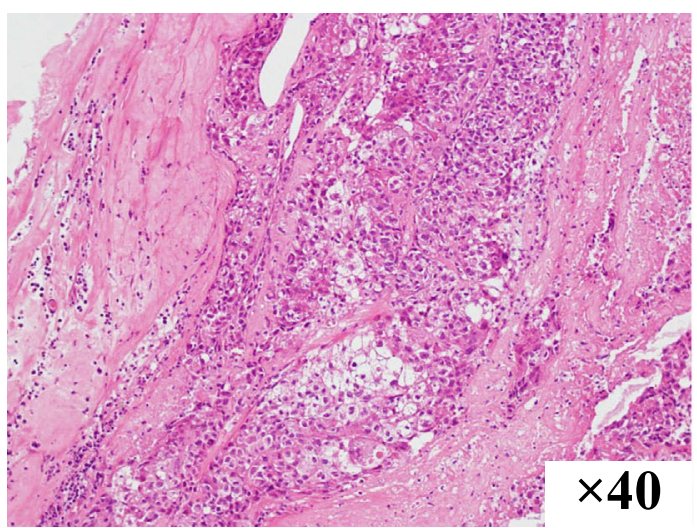

(B)

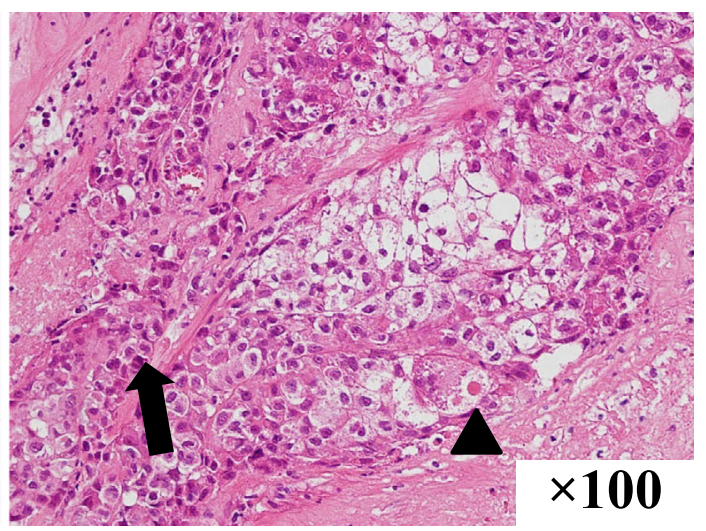

Figure 4. The histology of the right atrial tumor. The specimen had atypical cells with cord-like structures (arrow), globular hyaline bodies (arrow head), and clear cells. The tumor showed invasion into the right atrial endocardium. (A) Hematoxylin and Eosin (H\&E) staining (40-fold). (B) H\&E staining (100-fold).

(A)

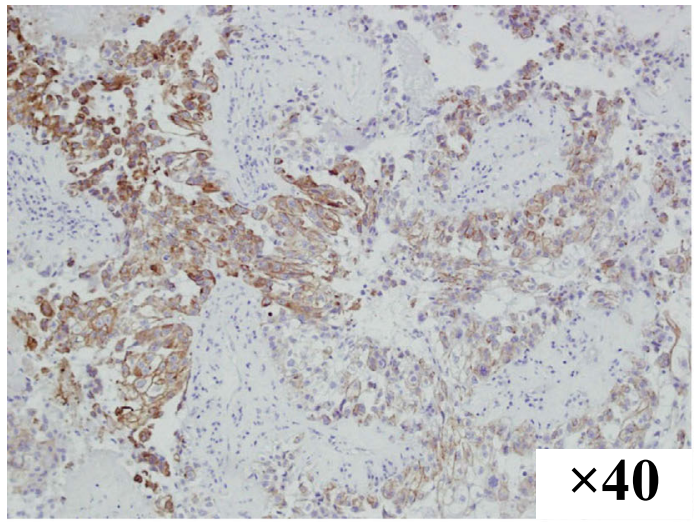

(C)

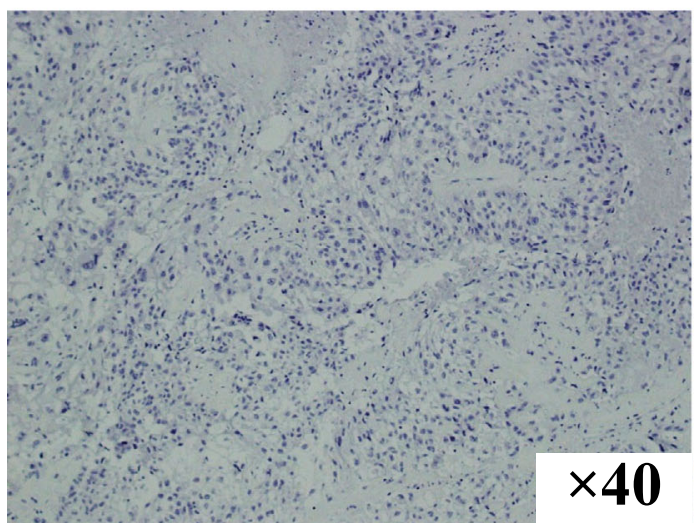

(B)

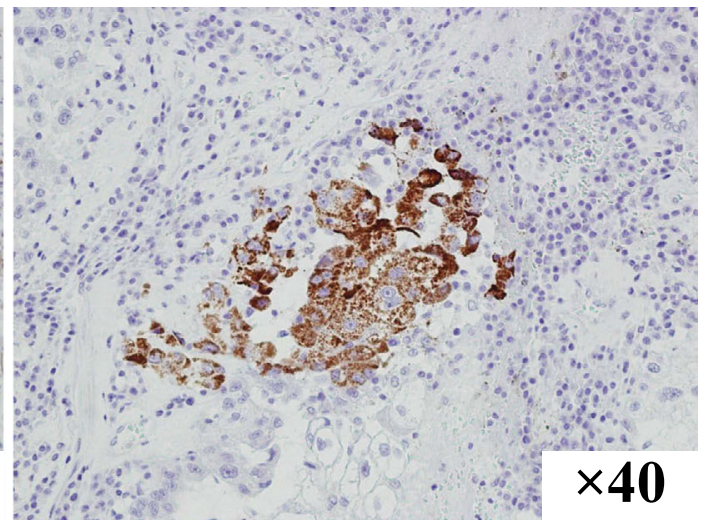

Figure 5. On immunohistological staining, CAM5.2 and HSA were positive, and AE1/AE3 was negative. (A) CAM5.2 immunohistochemical staining (40-fold). (B) HSA immunohistochemical staining (40-fold). (C) AE1/AE3 immunohistochemical staining (40-fold). CAM5.2: cytokeratin5.2, HSA: hepatocyte-specific antigen, AE1/AE3: cytokeratin-multi AE1/AE3

case, the right atrial tumor may have spread through the hepatic veins and IVC without tumor thrombus. As enhanced abdominal $\mathrm{CT}$ revealed that $\mathrm{HCC}$ in segment 6 had access to the intrahepatic vein (Fig. 2A), microinvasion to intrahe- patic HCC might have occurred.

Nakashima et al. reported that right atrial metastases are related to the differentiation of HCC, but whether or not they are related to liver cirrhosis is unknown. Many cases 
(88.3\%) exhibit extrahepatic metastases of HCC to the lungs, adrenal glands, bones, and peritoneum. The cases with right atrial metastases are more often diffuse-type than nodular- or massive-type HCC (2). Diffuse-type HCC tends to invade the portal and hepatic veins and may ultimately invade the right atrium and other organs. However, the present patient did not exhibit a diffuse-type HCC, and extrahepatic metastases, except for the right atrial metastasis, were absent. The histology of the atrial metastasis showed poorly differentiated HCC; we therefore suspected that the intrahepatic HCC also had high-grade malignancy. We believe that the etiology of the right atrial metastasis may have been microinvasion of HCC to the hepatic vein, although no invasion was detected on enhanced CT. This is the first report of HCC metastasis to the right atrium without intrahepatic vein invasion. A long-term survival is expected in patients treated for extrahepatic metastasis with good control of intrahepatic HCC.

The clinical symptoms, including respiratory distress, edema, and ascites, of right atrial metastasis are similar to those of right heart failure (14). Furthermore, sudden death has been reported (15) due to ball valve thrombus syndrome. To prevent sudden death due to this syndrome or pulmonary embolism, right atrial tumor resection is necessary. However, many cases are inoperable because of an advanced stage of cancer and a poor general condition. Some such cases have undergone TACE and/or RFA for intrahepatic HCC with chemoradiation therapy (CRT) (5). In their analysis of the survival benefit of surgical treatment for HCC with IVC and/or right atrial metastatic tumor, Wang et al. reported that the operation group (hepatectomy and thrombectomy) had a longer survival than the TACE group (TACE with or without CRT) (19 months vs. 4.5 months) (16). It was therefore believed that right atrial tumor resection not only prevented sudden death but also prolonged the survival. In our case, we believe that right atrial tumor resection and treatment for intrahepatic HCC resulted in a complete cure and a good survival.

This patient had undergone blood examinations and imaging inspection every few months. However, the right atrial tumor was not diagnosed until it had progressed. The diagnosis was delayed because the tumor was an isolated right atrial metastasis without hepatic vein invasion. In our case, the levels of tumor markers (PIVKA-II) were increased, and the patient underwent whole-body enhanced CT. Although we retrospectively checked the previous abdominal enhanced CT findings, we could not detect $\mathrm{HCC}$ or the right atrial tumor. Furthermore, the patient had never undergone MRI or echocardiography. High levels of PIVKA-II reportedly indicate a higher potential of biological malignancy than alphafetoprotein (AFP). HCC with high PIVKA-II levels sometimes shows vascular invasion or metastasis to other organs (17). We believe that the HCC of this patient had high potential for malignancy because of the high PIVKA-II levels. As a result, the HCC progressed rapidly. We should bear in mind that HCC patients may exhibit isolated right atrial metastases without hepatic vein invasion and that right atrial tumors should be resected to ensure the long-term survival.

\section{Conclusion}

This is the first report of an HCC patient with an isolated right atrial metastasis without hepatic vein invasion; the patient exhibited a prolonged survival after HCC treatment. This is an extremely rare case, and clinicians can benefit from our experience for future medical treatment.

The authors state that they have no Conflict of Interest (COI).

\section{References}

1. Okuda K. Hepatocellular carcinoma: clinicopathological aspects. J Gastroenterol Hepatol 12: S314-S318, 1997.

2. Nakashima T, Kojiro M, Kakizoe S, et al. Pathomorphological study on hepatocellular carcinoma - A study of tumor thrombi of inferior vena cava and right atrium. J Kurume Med Assoc 47: 722733, 1984.

3. Kojiro M, Nakahara H, Sugihara S, et al. Hepatocellular carcinoma with intra-atrial tumor growth. A clinicopathologic study of 18 autopsy cases. Arch Pathol Lab Med 108: 989-992, 1984.

4. Edmondson HA, Steiner PE. Primary carcinoma of the liver: a study of 100 cases among 48,900 necropsies. Cancer 7: 462-503, 1954.

5. Luo X, Zhang B, Dong S, Zhang B, Chen X. Hepatocellular carcinoma with tumor thrombus occupying the right atrium and portal vein: A case report and literature review. Medicine (Baltimore) 94: 34, 2015.

6. Sugano S, Takezawa K, Mizuyoshi H, et al. A case of hepatocellular carcinoma with intravascular tumor growth into right atrium. J Med Soc Toho 31: 216-222, 1984.

7. Reynen K. Frequency of primary tumors of the heart. Am J Cardiol 77: 107, 1996.

8. Al-Mamgani A, Baartman L, Baaijens M, et al. Cardiac metastases. Int J Clin Oncol 13: 369-372, 2008.

9. Mukai K, Shinkai T, Tominaga K, et al. The incidence of secondary tumors of the heart and pericardium: a 10-year study. Jpn J Clin Oncol 18: 195-201, 1988.

10. Marzouka GR, Badheka A, Rodriguez AP, et al. Hepatocellular carcinoma to the right ventricle. Case Rep Cardiol 2014: 192737, 2014.

11. Kuratomi S. A histopathological study of hepatocellular carcinoma-pathology of advanced hepatocellular carcinoma and intravascular tumor thrombosis in relation to vascular structure and alterations. Kanzo 17: 517-527, 1976.

12. Miyake M, Okudaira M, Hisauchi T. Pathology of liver disease from the vascular architectural point of view. Internal medicine 11: 604-612, 1963

13. Pesi B, Giudici F, Moraldi L, et al. Hepatocellular carcinoma on cirrhosis complicated with tumoral thrombi extended to the right atrium: results in three cases treated with major hepatectomy and thrombectomy under hypothermic cardiocirculatory arrest and literature review. World J Surg Oncol 14: 83, 2016.

14. Liu YC, Ho YL, Huang GT, et al. Clinical manifestations and survival of patients with hepatocellular carcinoma and cardiac metastasis. J Gastroenterol Hepatol 25: 150-155, 2010.

15. Sung AD, Cheng S, Moslehi J, et al. Hepatocellular carcinoma with intracavitary cardiac involvement: a case report and review of the literature. Am J Cardiol 102: 643-645, 2008.

16. Wang Y, Yuan L, Ge RL, et al. Survival benefit of surgical treatment for hepatocellular carcinoma with inferior vena cava/right atrium tumor thrombus: results of a retrospective cohort study. 
Ann Surg Oncol 20: 914-922, 2013.

17. Kiriyama S, Uchiyama K, Ueno M, et al. Triple positive tumor markers for hepatocellular carcinoma are useful predictors of poor survival. Ann Surg 254: 984-991, 2011.
The Internal Medicine is an Open Access article distributed under the Creative Commons Attribution-NonCommercial-NoDerivatives 4.0 International License. To view the details of this license, please visit (https://creativecommons.org/licenses/ by-nc-nd/4.0/).

(C) 2017 The Japanese Society of Internal Medicine Intern Med 56: 2589-2594, 2017 